Acknowledgments. We thank the leadership team at the Louis Stokes Cleveland VA Medical Center for their support of the Infection Control and Personnel Health departments during the pandemic.

Financial support. This work was supported by the Department of Veterans' Affairs.

Conflicts of interest. All authors report no conflicts of interest relevant to this article.

\section{References}

1. Jinadatha C, Jones LD, Choi H, et al. Transmission of SARS-CoV-2 in inpatient and outpatient settings in a Veterans' Affairs healthcare system. Open Forum Infect Dis 2021;8:ofab328.

2. Interim guidance for managing healthcare personnel with SARS-CoV-2 infection or exposure to SARS-CoV-2. Centers for Disease Control and Prevention website. https://www.cdc.gov/coronavirus/2019-ncov/yourhealth/quarantine-isolation.html. Accessed January 9, 2022.

3. Strategies to mitigate healthcare personnel staffing shortages. Centers for Disease Control and Prevention website. https://www.cdc.gov/coronavirus/ 2019-ncov/hcp/guidance-risk-assesment-hcp.html. Accessed January 9, 2022.

4. Cheng HY, Jian SW, Liu DP, et al. Contact tracing assessment of COVID-19 transmission dynamics in Taiwan and risk at different exposure periods before and after symptom onset. JAMA Intern Med 2020;180:1156-1163.

5. Ge Y, Martinez L, Sun S, et al. COVID-19 Transmission dynamics among close contacts of index patients with COVID-19: a population-based cohort study in Zhejiang Province, China. JAMA Intern Med 2021;181:1343-1350.

6. Pekosz A, Parvu V, Li M, et al. Antigen-based testing but not real-time polymerase chain reaction correlates with severe acute respiratory syndrome coronavirus 2 viral culture. Clin Infect Dis 2021;73:e2861-e2866.

7. Lee LYW, Rozmanowski S, Pang M, et al. SARS-CoV-2 infectivity by viral load, $S$ gene variants and demographic factors and the utility of lateral flow devices to prevent transmission. Clin Infect Dis 2021. doi: 10.1093/cid/ ciab421.

8. Pilarowski G, Lebel P, Sunshine S, et al. Performance characteristics of a rapid severe acute respiratory syndrome coronavirus 2 antigen detection assay at a public plaza testing site in San Francisco. J Infect Dis 2021;223: $1139-1144$.

9. Drain PK. Rapid diagnostic testing for SARS-CoV-2. N Engl J Med 2022;386:264-272.

\title{
Virus decay rates should not be used to reduce recommended room air clearance times
}

\author{
William G. Lindsley $\mathrm{PhD}^{1}$ (1), Stephen B. Martin Jr. PhD, $\mathrm{PE}^{1}\left(\mathbb{0}\right.$, Kenneth R. Mead PhD, $\mathrm{PE}^{2}$ (1) and \\ Duane R. Hammond MS, PE ${ }^{2}$ \\ ${ }^{1}$ National Institute for Occupational Safety and Health, Morgantown, West Virginia and ${ }^{2}$ National Institute for Occupational Safety and Health, Cincinnati, Ohio
}

To the Editors-We read with concern the letter by Hurlburt et $\mathrm{al}^{1}$ proposing revisions to the recommended room air clearance times for infectious aerosols in healthcare facilities. We believe that the calculations performed to justify the changes are based on flawed assumptions and an erroneous calculation. Experimental data on the survival of airborne SARS-CoV-2 virus and the dynamics of room ventilation do not support their conclusions.

Hurlburt et al based their proposed changes on data describing the effects of humidity on the viability of airborne influenza viruses, and on reports that influenza decays more rapidly at midrange humidities. They then assumed that these decay rates apply to SARS-CoV-2 as well. In fact, this is not the case. Schuit et $\mathrm{al}^{2}$ studied the decay in viability of airborne SARS-CoV-2 for relative humidities of $20 \%$ to $70 \%$ at $20^{\circ} \mathrm{C}$ and found that SARS-CoV-2 was relatively stable in air in the absence of sunlight $\left(\mathrm{k}_{\text {infect }}=0.008\right.$ per minute) and that humidity did not significantly affect the decay rate. Other researchers have also reported either no effect or a small effect of humidity on the decay rate of airborne SARS-CoV-2.,

Using data for influenza rather than SARS-CoV-2, Hurlburt et al assumed that a relative humidity of $40 \%$ to $60 \%$ would reduce the viability of SARS-CoV-2 by $30 \%$ to $50 \%$. Unfortunately, these researchers miscalculated the effect that this would have on air clearance times. They simply multiplied the equation for the

Author for correspondence: William G. Lindsley, E-mail: wlindsley@cdc.gov Cite this article: Lindsley WG, et al. (2022). Virus decay rates should not be used to reduce recommended room air clearance times. Infection Control \& Hospital Epidemiology, 43: 1987-1989, https://doi.org/10.1017/ice.2021.494 clearance time by their assumed reduction in viability, which has the mathematical effect of assuming that the reduction in viability occurs instantaneously. In fact, experimental data for SARS-CoV-2 and other viruses show that losses in viability are best modeled as an exponential decay. The correct version of the formula is

$$
t=\frac{-\ln [1-(P R E / 100)]}{A C H+\left(k_{\text {infect }} \times 60\right)} \times k_{\text {mix }} \times 60
$$

where PRE is the desired percent particulate removal (\%); $\mathrm{ACH}$ is the air exchange rate for the room ventilation (Air changes/ hour); $\mathrm{k}_{\text {infect }}$ is the decay constant for infectivity of the virus (per minute); $\mathrm{k}_{\mathrm{mix}}$ is the mixing factor (explained below); $\mathrm{t}$ is the time to achieve desired percent particle removal (minutes). The error in the authors' formula exaggerates the effect of losses in viability, especially over shorter times. The data from Schuit et $\mathrm{al}^{2}$ suggest that it would take 45 minutes for airborne SARSCoV-2 to lose $30 \%$ of its viability and 87 minutes to lose $50 \%$ of its viability, which is very different from the authors' assumption.

A second problem is that Hurlburt et al failed to include ventilation mixing factors in their calculations. The time required to remove airborne particles from a space can be estimated using the Centers for Disease Control and Prevention (CDC) Guidelines for Environmental Infection Control in Health Care Facilities (Table B.1). ${ }^{5}$ Table B.1 matches the values in the "none" column of figure 1 of the Hurlburt et al letter. However, Table B.1 assumes that the air in 
Table 1. Time Required for the Concentration of Viable Airborne virus in a Room to be Reduced by 95\% Using Different Assumptions for the Virus Decay Rate and the Room Mixing Factor ${ }^{a}$

\begin{tabular}{|c|c|c|c|c|}
\hline $\begin{array}{l}\text { Virus Viability Decay Constant } \\
\text { (per min) }\end{array}$ & No Decay & 0.008 & No Decay & 0.008 \\
\hline Room mixing factor & 1 & 1 & 3 & 3 \\
\hline Air changes/hour & \multicolumn{4}{|c|}{ Required Time, min } \\
\hline 1 & 180 & 121 & 539 & 364 \\
\hline 2 & 90 & 72 & 270 & 217 \\
\hline 3 & 60 & 52 & 180 & 155 \\
\hline 4 & 45 & 40 & 135 & 120 \\
\hline 5 & 36 & 33 & 108 & 98 \\
\hline 6 & 30 & 28 & 90 & 83 \\
\hline 7 & 26 & 24 & 77 & 72 \\
\hline 8 & 22 & 21 & 67 & 64 \\
\hline 9 & 20 & 19 & 60 & 57 \\
\hline 10 & 18 & 17 & 54 & 51 \\
\hline 11 & 16 & 16 & 49 & 47 \\
\hline 12 & 15 & 14 & 45 & 43 \\
\hline 13 & 14 & 13 & 41 & 40 \\
\hline 14 & 13 & 12 & 39 & 37 \\
\hline 15 & 12 & 12 & 36 & 35 \\
\hline 16 & 11 & 11 & 34 & 33 \\
\hline 17 & 11 & 10 & 32 & 31 \\
\hline 18 & 10 & 10 & 30 & 29 \\
\hline 19 & 9 & 9 & 28 & 28 \\
\hline 20 & 9 & 9 & 27 & 26 \\
\hline 21 & 9 & 8 & 26 & 25 \\
\hline 22 & 8 & 8 & 25 & 24 \\
\hline 23 & 8 & 8 & 23 & 23 \\
\hline 24 & 7 & 7 & 22 & 22 \\
\hline 25 & 7 & 7 & 22 & 21 \\
\hline 26 & 7 & 7 & 21 & 20 \\
\hline 27 & 7 & 7 & 20 & 20 \\
\hline 28 & 6 & 6 & 19 & 19 \\
\hline 29 & 6 & 6 & 19 & 18 \\
\hline 30 & 6 & 6 & 18 & 18 \\
\hline
\end{tabular}

${ }^{a}$ The room clearance time including the virus decay are included only to demonstrate that the effects of including experimental values for SARS-CoV-2 virus decay are small. Virus decay rates should not be included in real-world applications of room clearance time calculations because of the large uncertainties in decay rates.

the room is completely mixed; it is purely a mathematical estimate of room air dilution under ideal conditions. The footnotes to Table B.1 note that "The times given assume perfect mixing of the air within the space (ie, mixing factor $=1$ ). However, perfect mixing usually does not occur. Removal times will be longer in rooms or areas with imperfect mixing or air stagnation." Thus, the appropriate use of Table B.1 to establish clearance times requires multiplying the times in the table by a mixing factor $(\mathrm{k})$ that ranges between 1 and $10 .^{6,7}$ This factor represents how well the ventilation system mixes and dilutes the concentration of airborne particles within the room. ${ }^{8}$ It can be experimentally determined for a specific room, or, as a rule of thumb, a mixing factor of $k=3$ is often applied to rooms with higher airflow rates $(\geq 6 \mathrm{ACH})$ and good placement of supply and exhaust grilles. In that case, the time identified in Table B.1 would be multiplied by 3 to estimate the clearance time prior to re-entry.

The corrected times estimated to reduce the concentration of viable airborne virus in a room by $95 \%$ are shown in Table 1 in this letter. For a $95 \%$ concentration reduction at an air change rate of $6 \mathrm{ACH}$ and using the decay coefficient for SARS-CoV-2 from Schuit et $\mathrm{al},{ }^{2}$ the room clearance time is only reduced by 2 minutes, from 30 to 28 minutes. This is very different from the authors' predicted 20- and 15-minute clearance times that assume immediate $30 \%$ and $50 \%$ reductions in viability, respectively. Table 1 further demonstrates that including the mixing factor has a large impact on the clearance time.

Finally, the decay in viability of SARS-CoV-2 (and airborne viruses in general) varies substantially depending upon the strain of the virus, the composition of the suspending medium, the air temperature, the presence of sunlight, and other factors. ${ }^{2-4,9,10}$ Much remains to be learned about the stability of airborne viruses. Prudence dictates that adjustments to room clearance times are not made based on assumptions about virus viability until this phenomenon is better understood.

In conclusion, the modifications to the calculation of room air clearance times proposed by the authors are not supported by current scientific evidence. Near the end of the letter, the authors write, "The interaction between viruses and relative humidity is complex, and large knowledge gaps exist." We agree wholeheartedly with this statement, and it serves as an excellent argument against the proposed reductions in room air clearance times until the stability and decay in viability of airborne viruses are better understood.

Acknowledgments. The findings and conclusions in this report are those of the authors and do not necessarily represent the official position of the National Institute for Occupational Safety and Health (NIOSH) of the Centers for Disease Control and Prevention (CDC).

Financial support. No financial support was provided relevant to this article.

Conflicts of interest. All authors report no conflicts of interest relevant to this article.

\section{References}

1. Hurlburt AW, DeKleer RJ and Bryce EA. Clearing the air: Addressing air clearance times for infectious aerosols in healthcare facilities. Infect Control Hosp Epidemiol 2021;42:1143-1144.

2. Schuit M, Ratnesar-Shumate S, Yolitz J, et al. Airborne SARS-CoV-2 is rapidly inactivated by simulated sunlight. J Infect Dis 2020;222:564-571.

3. Dabisch P, Schuit M, Herzog A, et al. The influence of temperature, humidity, and simulated sunlight on the infectivity of SARS-CoV-2 in aerosols. Aerosol Sci Technol 2020;55:142-153.

4. Smither SJ, Eastaugh LS, Findlay JS and Lever MS. Experimental aerosol survival of SARS-CoV-2 in artificial saliva and tissue culture media at medium and high humidity. Emerg Microbes Infect 2020;9:1415-1417.

5. Guidelines for environmental infection control in healthcare facilities Recommendations of $\mathrm{CDC}$ and the Healthcare Infection Control Practices Advisory Committee (HICPAC). Centers for Disease Control and Prevention website. https://www.cdc.gov/infectioncontrol/guidelines/ environmental/index.html. Published 2003. Accessed October 21, 2021.

6. Wabeke RL. Air Contaminants and Industrial Hygiene Ventilation : A Handbook of Practical Calculations, Problems, and Solutions. Boca Raton, FL: Lewis; 1998. 
7. Bozzi CJ, Burwen DR, Dooley SW, et al. Guidelines for preventing the transmission of Mycobacterium tuberculosis in healthcare facilities, 1994. Morb Mortal Wkly Rep 1994;43(RR-13):1-132.

8. Tuberculosis infection control: a pratical manual for preventing TB. Curry International Tuberculosis Center University of California, San Francisco website. https://www.currytbcenter.ucsf.edu/products/tuberculosis-infectioncontrol-practical-manual-preventing-tb. Published 2011. Accessed December $5,2021$.
9. van Doremalen N, Bushmaker T, Morris DH, et al. Aerosol and surface stability of SARS-CoV-2 as compared with SARS-CoV-1. N Engl J Med 2020; 382:1564-1567.

10. Fears AC, Klimstra WB, Duprex P, et al. Persistence of severe acute respiratory syndrome coronavirus 2 in aerosol suspensions. Emerg Infect Dis 2020;26:2168-2171.

\title{
Disproportionate distribution of coronavirus disease 2019 (COVID-19) antiviral pills: Vaccine inequity replay?
}

\author{
Taha Gul Shaikh MBBS ${ }^{1}$ (1), Summaiyya Waseem MBBS $^{1}$ (1) , Syed Hassan Ahmed MBBS ${ }^{1}$, \\ Muhammad Sohaib Asghar MBBS ${ }^{2}$ (1) and Muhammad Junaid Tahir MBBS ${ }^{3}$ (1) \\ ${ }^{1}$ Dow University of Health Sciences, Karachi, Pakistan, ${ }^{2}$ Dow University of Health Sciences-Ojha Campus, Karachi, Pakistan and ${ }^{3}$ Lahore General Hospital, \\ Lahore, Pakistan
}

To the Editor-The rapid development of the coronavirus disease 2019 (COVID-19) vaccine was only possible due to massive international collaboration in the research and development sector. However, the disproportionate distribution of vaccines led to COVID-19 hotspots and the emergence of new variants, which has already prolonged the pandemic. ${ }^{1}$ Since the beginning, the World Health Organization (WHO) has demanded equitable distribution of vaccines. To accomplish this, an initiative named COVID-19 Vaccine Global Access (COVAX) was started to equally distribute vaccines among all countries regardless of their contribution to the development. ${ }^{2}$ However, did not occur because affluent countries, to quickly vaccinate their population, started to stockpile the vaccines. Thus, the distribution was asymmetrically in their favor, leading to a global shortage, especially in the third world and developing countries. Recently, US President Joe Biden stated in a vaccine summit that the United States would distribute the 100 million stockpiled vaccines to the lower- and middleincome countries (LMICs). ${ }^{3}$ This extensive stockpile exemplifies the hoarding that occurred, which resulted in untold unnecessary loss of lives. According to a model by Northeastern University, the proportional distribution of vaccines can avoid twice as many deaths as vaccine distribution limited to high-income countries. ${ }^{4}$ To further illustrate this issue, a Lorenz curve and Gini coefficients, which are used for inequality indices, were adopted. ${ }^{5}$ The Lorenz curve suggests that $20 \%$ of the world population had control $>95 \%$ of COVID-19 vaccinations. Similarly, the Gini coefficient for vaccines, ranging from 0 to 1 , was 0.86 , which indicates highly unequal distribution. ${ }^{1}$ According to the Global Dashboard of vaccine equity, only $3.07 \%$ of people have been vaccinated for severe acute respiratory syndrome coronavirus 2 (SARS-CoV-2) in LMICs compared to $60.18 \%$ in high-income countries (HICs) as of September $15,2021 .^{6}$ This situation has been further aggravated by ineffective and selective government policies. In Brazil, only people living in legally marked territories were vaccinated, leaving others

Author for correspondence: Dr Muhammad Sohaib Asghar, E-mail: sohaib_asghar123@yahoo.com

Cite this article: Shaikh TG, et al. (2022). Disproportionate distribution of coronavirus disease 2019 (COVID-19) antiviral pills: Vaccine inequity replay?. Infection Control \& Hospital Epidemiology, 43: 1989-1990, https://doi.org/10.1017/ice.2021.532 unvaccinated. ${ }^{7}$ In India, inadequate and inequitable vaccine distribution has led to several instances of vaccine shortage despite this country being the top manufacturer of COVID-19 vaccines. ${ }^{8}$ The unequal distribution will not only aggravate the pandemic but will also increase inequality and deepen the gap between different segments of society. Ultimately, this will reverse the progress of human development. ${ }^{9}$ The situation of unequal drug distribution is not limited to COVID-19 vaccines. African countries have been severely affected by the ongoing crisis: pre-existing drug shortages have been worsened due to the effect of the pandemic on the global supply chain. In Nigeria alone, $70 \%$ of the drugs are imported, but due to global shortage and lockdowns, essential and life-saving drugs, including antiviral and antiretroviral drugs, have become scarce. ${ }^{10}$

During this pandemic, we have seen a remarkable pace and progress in terms of COVID-19 vaccination. Lately, 2 pharmaceutical companies, Pfizer and Merck, have announced the development of COVID-19 antiviral pills that significantly decrease hospitalizations. This discovery is a blessing for those countries where there is a shortage of vaccines. ${ }^{11}$ It is too early to predict whether these drugs will meet expectations. In theory, the drugs should be effective against the current variants including the highly transmissible and aggressive $\delta$ (delta) variant. The disease burden in affected areas should be a properly assessed, which is a complex process. A strategy based on the egalitarian concept of distribution should be used that emphasizes the equality of every individual concerning health and well-being. ${ }^{9}$ This equity could be achieved by effective distribution based on a well-designed system of distributive justice. ${ }^{9}$ At times, distribution is not easy given the geographical conditions and lack of facilities to store and transport these medicines, but the efforts of Nepal in eradicating tuberculosis are remarkable given that most of its area is mountainous and hilly. ${ }^{12}$ Another possibility of unfair COVID-19 pill distribution could relate to wealthy countries paying a handsome amount to these companies, leaving little to no room for LMICs. In most developing countries, it is more profitable for companies to sell drugs to the wealthy segment of the society instead of selling to a larger number of people at lower prices. As a result, medicines remain inaccessible to most of the population. To prevent this from happening 